\title{
On the second largest eigenvalue of some Cayley graphs of the symmetric group
}

\author{
Johannes Siemons ${ }^{1}$ (D) Alexandre Zalesski ${ }^{2}$
}

Received: 2 March 2021 / Accepted: 29 September 2021 / Published online: 10 November 2021

(C) The Author(s) 2021

\section{Abstract}

Let $S_{n}$ and $A_{n}$ denote the symmetric and alternating group on the set $\{1, \ldots, n\}$, respectively. In this paper we are interested in the second largest eigenvalue $\lambda_{2}(\Gamma)$ of the Cayley graph $\Gamma=\operatorname{Cay}(G, H)$ over $G=S_{n}$ or $A_{n}$ for certain connecting sets $H$. Let $1<k \leq n$ and denote the set of all $k$-cycles in $S_{n}$ by $C(n, k)$. For $H=C(n, n)$ we prove that $\lambda_{2}(\Gamma)=(n-2)$ ! (when $n$ is even) and $\lambda_{2}(\Gamma)=2(n-3)$ ! (when $n$ is odd). Further, for $H=C(n, n-1)$ we have $\lambda_{2}(\Gamma)=3(n-3)(n-5)$ ! (when $n$ is even) and $\lambda_{2}(\Gamma)=2(n-2)(n-5)$ ! (when $n$ is odd). The case $H=C(n, 3)$ has been considered in Huang and Huang (J Algebraic Combin 50:99-111, 2019). Let $1 \leq r<k<n$ and let $C(n, k ; r) \subseteq C(n, k)$ be set of all $k$-cycles in $S_{n}$ which move all the points in the set $\{1,2, \ldots, r\}$. That is to say, $g=\left(i_{1}, i_{2}, \ldots, i_{k}\right)\left(i_{k+1}\right) \ldots\left(i_{n}\right) \in C(n, k ; r)$ if and only if $\{1,2, \ldots, r\} \subset\left\{i_{1}, i_{2}, \ldots, i_{k}\right\}$. Our main result concerns $\lambda_{2}(\Gamma)$, where $\Gamma=\operatorname{Cay}(G, H)$ with $H=C(n, k ; r)$ with $1 \leq r<k<n$ when $G=S_{n}$ if $k$ is even and $G=A_{n}$ if $k$ is odd. Here we observe that

$$
\lambda_{2}(\Gamma) \geq(k-2) !\left(\begin{array}{l}
n-r \\
k-r
\end{array}\right) \frac{1}{n-r}\left((k-1)(n-k)-\frac{(k-r-1)(k-r)}{n-r-1}\right) .
$$

We prove that this bound is attained in the special case $k=r+1$, giving $\lambda_{2}(\Gamma)=$ $r !(n-r-1)$. The cases with $H=C(n, 3 ; 1)$ and $H=C(n, 3 ; 2)$ were considered earlier in [6].

Dedicated to the memory of Gordon James.

$凶$ Johannes Siemons

J.Siemons@uea.ac.uk

Alexandre Zalesski

alexandre.zalesski@gmail.com

1 School of Mathematics, University of East Anglia, University Plain, Norwich, NR47TJ, UK

2 Department of Physics, Mathematics and Informatics, National Academy of Sciences of Belarus, 66 Prospekt Nezavisimosti, Minsk, Belarus 
Keywords Symmetric group · Cayley graph · Adjacency matrix · Eigenvalues · Weyl Inequality

Mathematics Subject Classification 20G05 · 20G40

\section{Introduction}

Let $\Gamma$ be a finite undirected graph and let $A$ denote its adjacency matrix. The eigenvalues of this matrix, together with their multiplicities, are an important invariant of the graph. Since $A$ is symmetric, all eigenvalues are real. For a regular graph of degree $d$, it is well-known that the largest eigenvalue of $A$ is $\lambda_{1}(\Gamma)=d$. The second largest eigenvalue, denoted by $\lambda_{2}(\Gamma)$, plays an important role in many theoretical and practical applications of graph theory, from geometry to computer science. The literature on the second largest eigenvalue is extensive, overviews and further references can be found in $[1,3,5,12,13,16]$. For regular graphs, the spectral gap $\lambda_{1}(\Gamma)-\lambda_{2}(\Gamma)$ is known as the algebraic connectivity of $\Gamma$.

In this paper we are concerned with the second largest eigenvalue of certain Cayley graphs $\operatorname{Cay}(G, H)$ where $G$ is a symmetric or alternating group and where $H$ a connecting set in $G$. (In particular, we have $H=H^{-1}=\left\{h^{-1} \mid h \in H\right\}$, the identity element of $G$ does not belong to $H$, and $H$ is not contained in any proper subgroup of $G$.) For the definition of Cayley graphs see Sect. 2.4. References to recent work on the second largest eigenvalue of Cayley graphs over symmetric groups can be found in [6]. Even for symmetric groups, an explicit computation of it appears to be one of the most challenging problem in the area.

To state our results, let $S_{n}=\operatorname{Sym}(1, \ldots, n)$ and $A_{n}$ be the symmetric and alternating group on the set $\{1, \ldots, n\}$, respectively. For $1<k \leq n$, a $k$-cycle in $S_{n}$ is a permutation of the shape $g=\left(i_{1}, i_{2}, \ldots, i_{k}\right)\left(i_{k+1}\right) \ldots\left(i_{n}\right)$ with $\left|\left\{i_{1}, i_{2}, \ldots, i_{k}\right\}\right|=k$. The set of all $k$-cycles in $S_{n}$ is denoted by $C(n, k)$.

Theorem 1.1 Let $\Gamma=\operatorname{Cay}(G, H)$ with $H=C(n, n)$ for $n>4$ and $G=S_{n}$ when $n$ is even and $G=A_{n}$ when $n$ is odd. Then $\lambda_{1}(\Gamma)=(n-1)$ !. Furthermore, $\lambda_{2}(\Gamma)=$ $(n-2)$ ! when $n$ is even and $\lambda_{2}(\Gamma)=2(n-3)$ ! when $n$ is odd.

Theorem 1.2 Let $\Gamma=\operatorname{Cay}(G, H)$ with $H=C(n, n-1)$ for $n>4$ and $G=S_{n}$ when $n$ is odd and $G=A_{n}$ when $n$ is even. Then $\lambda_{1}(\Gamma)=n(n-2)$ !. Furthermore, $\lambda_{2}(\Gamma)=3(n-3)(n-5)$ ! when $n$ is even and $\lambda_{2}(\Gamma)=2(n-2)(n-4)$ ! when $n$ is odd.

An essential feature of the Cayley graphs in these two theorems is that the connecting set $H$ is invariant under conjugation by $G$. In fact, $C(n, k)$ is a conjugacy class of $S_{n}$. If $k=n$ or $n-1$, the above results are deduced from some analysis of the group characters in a relatively straightforward way. For arbitrary $k$, computing the second largest eigenvalue for $\operatorname{Cay}(G, C(n, k))$ is an open problem. For $k=3$ this is solved in [6, Theorem 3.4], for $k=2$ see [10, Lemma 3].

Next let $1 \leq r<k<n$ and let $C(n, k ; r) \subseteq C(n, k)$ be set of all $k$-cycles in $\operatorname{Sym}(1, \ldots, n)$ which move all the points in the set $\{1,2, \ldots, r\}$. That is to 
say, $g=\left(i_{1}, i_{2}, \ldots, i_{k}\right)\left(i_{k+1}\right) \ldots\left(i_{n}\right) \in C(n, k ; r)$ if and only if $\{1,2, \ldots, r\} \subset$ $\left\{i_{1}, i_{2}, \ldots, i_{k}\right\}$. Clearly, $|C(n, k ; r)|=(k-1) !\left(\begin{array}{c}n-r \\ k-r\end{array}\right)$.

Our main results are Theorems 1.3 and 1.4. As $\lambda_{1}(\Gamma)=|H|$ in general (whenever $H$ is a connecting set), we focus on $\lambda_{2}(\Gamma)$.

Theorem 1.3 Let $n>4$ be an integer and $1 \leq r<k<n$. Let $\Gamma=\operatorname{Cay}(G, H)$ with $H=C(n, k ; r)$, where $G=S_{n}$ when $k$ is even and $G=A_{n}$ when $k$ is odd. Then $\mu_{2}=(k-2) !\left(\begin{array}{c}n-r \\ k-r\end{array}\right) \frac{1}{n-r}\left((k-1)(n-k)-\frac{(k-r-1)(k-r)}{n-r-1}\right)$ is an eigenvalue of $\Gamma$ and hence $\lambda_{2} \geq \mu_{2}$.

We conjecture that $\lambda_{2}=\mu_{2}$ for any $r, k$ with $1 \leq r<k<n$. We prove this conjecture for $k=r+1$ for arbitrary $r$ with $1 \leq r<n-1$ :

Theorem 1.4 Let $n>4$ be an integer and $2 \leq r \leq n-2$. Let $\Gamma=\operatorname{Cay}(G, H)$ with $H=C(n, r+1 ; r)$ where $G=S_{n}$ when $r$ is odd and $G=A_{n}$ when $r$ is even. Then $\lambda_{1}(\Gamma)=r !(n-r)$ and $\lambda_{2}(\Gamma)=r !(n-r-1)$.

In Theorems 1.3 and 1.4, in contrast to Theorems 1.1 and 1.3, the connecting set $H$ is not invariant under $S_{n}$. However, we make essential use of the fact that $H$ is invariant under a subgroup isomorphic to $S_{r} \times S_{n-r}$. Other cases where the bound is sharp include $\Gamma=\operatorname{Cay}(G, H)$ with $H=C(n, 3 ; 1)$ where $\lambda_{2}(\Gamma)=n^{2}-5 n+5$ by $[6$, Theorems 3.2]. The cases $H=C(n, 3 ; 2)$ with $\lambda_{2}(\Gamma)=2 n-6$ and $H=C(n, 2 ; 1)$ with $\lambda_{2}(\Gamma)=n-2$ were resolved earlier in [6, Theorem 2.3] and [12, Section 8.1], respectively.

Notation: All groups considered here are finite. Further, all modules and representations are over the field $\mathbb{C}$ of complex numbers. By $\mathbb{C} G$ we denote the group algebra of the group $G$ over $\mathbb{C}$. If $H \subset G$, then $|H|$ is the number of elements of $H$ and $H^{+}$ is the sum of all $h \in H$, as an element of $\mathbb{C} G$. A sentence such as ' $L$ is a $G$-module' means that $L$ is a $\mathbb{C} G$-module. If $L$ is a $\mathbb{C} G$-module and $X$ is a subgroup of $G$, we write $\left.L\right|_{X}$ for the restriction of $L$ to $X$. The trivial $G$-module and its character are denoted by $1_{G}$. We assume that the reader is familiar with general notions and elementary facts of finite group theory, including the representation theory of $S_{n}$ and $A_{n}$.

\section{Preliminaries}

We collect several well-known facts and prerequisites for the paper. All graphs are finite, undirected and without multiple edges.

\subsection{Graphs and eigenvalues}

Let $\Gamma$ be a graph on the vertex set $V$; we put $m=|V|$. For $u, v \in V$, we write $u \sim v$ if $u$ is adjacent to $v$. For $v \in V$, the set $N(v)=\{u \in V: u \sim v\}$ denotes the set of neighbors of $v$ and $d(v)=|N(v)|$ is the degree of $v$. The graph is regular of degree $d$ if $d(v)=d$ for all $v \in V$. A permutation $\gamma$ of $V, u \mapsto u^{\gamma}$, is an automorphism of $\Gamma$ if $(N(v))^{\gamma}=N\left(v^{\gamma}\right)$ for all $v \in V$. The group of all automorphisms of $\Gamma$ is denoted by $\operatorname{Aut}(\Gamma)$. 
The adjacency matrix $A=\left(a_{u, v}\right)$ of $\Gamma$ is given by $a_{u, v}=1$ if $u \sim v$ and $a_{u, v}=0$ otherwise. Then $A$ determines $\Gamma$. The eigenvalues of $\Gamma$ are, by definition, the eigenvalues of $A$. These are real since $A$ is symmetric.

Theorem 2.1 [11, Proposition 1.48] Let $\Gamma$ be a regular graph of degree $d$ and let $A$ be its adjacency matrix. Then

(1) $d$ is an eigenvalue of A. Furthermore, $d$ has multiplicity 1 if and only if $\Gamma$ is connected;

(2) $\Gamma$ is bipartite if and only if $-d$ is an eigenvalue of $A$;

(3) $d \geq|\lambda|$ for all eigenvalues $\lambda$ of $A$. If $\Gamma$ is bipartite and if $\lambda$ is an eigenvalue of $A$, then $-\lambda$ is an eigenvalue of $A$ with the same multiplicity as $\lambda$.

\subsection{Equitable partitions}

Let $\Gamma$ be a graph with vertex set $V$. Let $P$ be a partition $V=V_{1} \cup \cdots \cup V_{k}$ of $V$. Then $P$ is called equitable if $\left|N\left(v_{i}\right) \cap V_{j}\right|$ does not depend on the choice of $v_{i} \in V_{i}$ for all $1 \leq i, j \leq k$. If $P$ is equitable, then the matrix $B=B(P)=\left(\left|V_{i} \cap N\left(v_{j}\right)\right|\right)$ with $1 \leq i, j \leq k$ is called the associated matrix of $P$. As usual, a second partition $P^{\prime}$ is a refinement of $P$ if every class of $P$ is some union of classes of $P^{\prime}$.

For any graph $\Gamma$ examples of equitable partitions arise from the orbits of a group of automorphisms of $\Gamma$, see Lemma 2.3. For this reason, equitable partitions are also called generalized orbits. For incidence graphs of geometries also the term tactical decomposition is used, see Dembowski's book [4].

Let $\mathbb{C} V$ be the vector space with basis $V$ over $\mathbb{C}$. Then $A$ can be viewed as a linear transformation of $\mathbb{C} V$, and then $A v=\sum_{x \in N(v)} x$ for every $v \in V$. Let $(\cdot, \cdot)$ be the standard bilinear form on $\mathbb{C} V$ defined by $(u, v)=\delta_{u, v}$ (the Kronecker delta) for $u, v \in V$. Let $V=V_{1} \cup \cdots \cup V_{k}$ be a partition $P$ of $V$. Set $w_{i}=\sum_{u \in V_{i}} u$ for $i=1, \ldots, k$. Then $\left(w_{i}, A v\right)=\left|N(v) \cap V_{i}\right|$ (as $\left(w_{i}, u\right)=1$ for $u \in V$ if and only if $u \in V_{i}$ ). Let $\mathbb{C} P$ be the vector space with basis $w_{1}, \ldots, w_{k}$. In this notation we have:

Lemma 2.2 Let $\Gamma$ be a graph with vertex set $V$ and adjacency matrix $A$, and let $P$ be a partition $V=V_{1} \cup \cdots \cup V_{k}$ of $V$. Then $P$ is equitable if and only if $\mathbb{C} P$ is $A$-invariant (that is, $A x \in \mathbb{C} P$ for all $\mathrm{x} \in \mathbb{C} P$ ). Furthermore, if $P$ is equitable then $B(P)$ is the matrix of the restriction of $A$ to $\mathbb{C} P$ for the basis $w_{1}, \ldots, w_{k}$. In particular, all eigenvalues of $B$ are eigenvalues of $\Gamma$. If $\Gamma$ is regular of degree $d$, then $d$ is an eigenvalue of $B$.

In addition, if $P^{\prime}$ is an equitable refinement of $P$ then the eigenvalues of $B(P)$ are eigenvalues of $B\left(P^{\prime}\right)$.

Proof For $i=1, \ldots, k$, we have

$$
A w_{i}=\sum_{v \in V}\left(A w_{i}, v\right) v=\sum_{v \in V}\left(w_{i}, A v\right) v=\sum_{v \in V}\left(w_{i}, \sum_{u \in N(v)} u\right) v=\sum_{v \in V}\left|N(v) \cap V_{i}\right| v
$$


To be in $\mathbb{C} P$, the right-hand side must be constant on every $V_{j}$, that is, $\left|N(v) \cap V_{i}\right|=$ $\left|N\left(v^{\prime}\right) \cap V_{i}\right|$ for every $j \in\{1, \ldots, k\}$ and every $v, v^{\prime} \in V_{j}$. The converse and the second assertion of the lemma follows from (1) as well. Finally, if $\Gamma$ is regular of degree $d$ then $A\left(w_{1}+\cdots+w_{k}\right)=A \sum_{v \in V} v=d \sum_{v \in V} v=d\left(w_{1}+\cdots+w_{k}\right)$, so $d$ is an eigenvalue of $A$ on $\mathbb{C} P$.

Let $V=V_{1}^{\prime} \cup \cdots \cup V_{l}^{\prime}$ be a refinement $P^{\prime}$ of $P$ and let as above $w_{1}^{\prime}, \ldots, w_{l}^{\prime}$ be a basis of $\mathbb{C} P^{\prime}$. Then every $w_{j}(j=1, \ldots, k)$ is a linear combination of $w_{1}^{\prime}, \ldots, w_{l}^{\prime}$, so $\mathbb{C} P \subset \mathbb{C} P^{\prime}$, whence the additional statement.

Lemma 2.3 Let $\Gamma$ be a graph and $G$ a group of automorphisms of $\Gamma$. Let $V_{1}, \ldots, V_{k}$ be the orbits of $G$ on the vertex set $V$ of $\Gamma$. Then $V=V_{1} \cup \cdots \cup V_{k}$ is equitable.

Proof For $i, j$ with $1 \leq i, j \leq k$ let $v, v^{\prime} \in V_{i}$. Then there exists $g \in G$ so that $g(v)=v^{\prime}$ and therefore $\left.\left|N(v) \cap V_{j}\right|=\left|g\left(N(v) \cap V_{j}\right)\right|=\mid N(g(v)) \cap g\left(V_{j}\right)\right) \mid=$ $\left|N\left(v^{\prime}\right) \cap V_{j}\right|$.

\subsection{Eigenvalue inequalities}

We denote the distinct eigenvalues of a graph $\Gamma$ by

$$
\lambda_{1}>\lambda_{2}>\cdots>\lambda_{m^{\prime}}
$$

for some $m^{\prime} \leq m$. If $\Gamma$ is regular of degree $d$, then $\lambda_{1}=d$ by Theorem 2.1. The second largest eigenvalue, $\lambda_{2}$, plays a distinguished role in graph theory as it provides bounds for the isoperimetric constant of $\Gamma$, see also graph expanders, the Kahzdan constant and Cheeger inequality in [11]. From Theorem 2.1 and Lemma 2.2, we immediately have

Lemma 2.4 Let $P$ be an equitable partition of the regular graph $\Gamma$ and let $\beta_{2}$ be the second largest eigenvalue of $B(P)$. Then $\lambda_{2} \geq \beta_{2}$.

Upper and lower bounds on eigenvalues of a symmetric matrix over the reals can be obtained from a theorem of Hermann Weyl [18], see also [3, Theorem 2.8.1] and [2].

Theorem 2.5 (Weyl Inequality) Let $C=A+B$ be symmetric $(m \times m)$-matrices, and let $\gamma_{1} \geq \cdots \geq \gamma_{m}, \alpha_{1} \geq \cdots \geq \alpha_{m}, \beta_{1} \geq \cdots \geq \beta_{m}$ be the eigenvalues of $C, B, A$, respectively. Then for $1 \leq i, j \leq m$, we have

$$
\gamma_{i+j-1} \leq \alpha_{i}+\beta_{j} \text { whenever } i+j-1 \leq m
$$

and

$$
\gamma_{i+j-m} \geq \alpha_{i}+\beta_{j} \text { whenever } i+j-1 \geq m \text {. }
$$

We note in particular, for $i, j \in\{1,2\}$ we have

$$
\gamma_{2} \leq \alpha_{2}+\beta_{1} \text { and } \gamma_{2} \leq \alpha_{1}+\beta_{2}
$$


We refer to (4) as the Weyl inequality.

For adjacency matrices, we have several applications. First let $\gamma_{1} \geq \gamma_{2} \geq \gamma_{3} \geq$ $\cdots \geq \gamma_{m}$ be all eigenvalues of $\Gamma$, with repetitions. If $\Gamma$ is regular of degree $d$ and connected, then we have $d=\gamma_{1}=\lambda_{1}>\lambda_{2}=\gamma_{2}$ by Theorem 2.1, for other eigenvalues repetitions may appear.

Let $v$ be a vertex of $\Gamma$ and let $\Gamma^{\prime}$ be obtained from $\Gamma$ by deleting all edges incident with $v$. Denote the adjacency matrix of $\Gamma^{\prime}$ by $A^{\prime}$ and let $\gamma_{1}^{\prime} \geq \gamma_{2}^{\prime} \geq \cdots \geq \gamma_{m}^{\prime}$ be all eigenvalues of $\Gamma^{\prime}$, with repetitions. Next let $\Sigma$ be the 'star' at $v$ with $d$ rays, where $d$ is the degree of $v$, and with $m-d-1$ isolated vertices. Denote its adjacency matrix by $S$. The eigenvalues of the star are $\sigma_{1}=\sqrt{d}>\sigma_{2}=0=\cdots=\sigma_{m-1}>\sigma_{m}=-\sqrt{d}$, by a simple computation. Then $A=A^{\prime}+S$ and from Theorem 2.5 , we obtain the following

Corollary 2.6 Let $v$ be a vertex of the connected graph $\Gamma$ and let $\Gamma^{\prime}$ be obtained by deleting all edges incident with $v$. Let $\lambda_{1}>\lambda_{2}$ and $\lambda_{1}^{\prime}>\lambda_{2}^{\prime}$ be the largest and second largest eigenvalues of $\Gamma$ and $\Gamma^{\prime}$, respectively. Then

$$
\lambda_{1} \geq \lambda_{1}^{\prime} \geq \lambda_{2} \geq \lambda_{2}^{\prime}
$$

Furthermore, if $d$ is the degree of $v$ then

$$
\left|\lambda_{i}-\lambda_{i}^{\prime}\right| \leq \sqrt{d}
$$

for $1 \leq i \leq 2$.

Accounting for the isolated vertex $v$, the spectrum of the graph $\Gamma[V \backslash\{v\}]$ induced on $V \backslash\{v\}$ is obtained from the spectrum of $\Gamma^{\prime}$ by removing one eigenvalue $\lambda^{\prime}=0$. The first part of the corollary is therefore an instance of the well-known interlacing theorem, see [3, Theorem 2.5.1]. However, the second part of the corollary appears to be new.

\subsection{Cayley graphs}

Let $G$ be a group with identity element 1 or $1_{G}$ and let $H$ be a subset of $G$. Then $H$ is a connecting set if (i) $1 \notin H$, (ii) $H=H^{-1}\left(=\left\{h^{-1} \mid h \in H\right\}\right)$ and (iii) $H$ is not contained in any proper subgroup of $G$. For such a set $H$, the Cayley graph $\operatorname{Cay}(G, H)$ is the graph with vertex set $V=G$ so that two vertices $u, v \in G$ are adjacent if and only if $u v^{-1} \in H$. Thus, $N(v)=H v$ and so $\operatorname{Cay}(G, H)$ is regular of degree $|H|$. The adjacency matrix of $\operatorname{Cay}(G, H)$ is denoted by $A=A(G, H)$.

More generally, one can construct a similar graph $\Gamma(G, H)$ if (i),(ii) holds and (iii) fails. Then $\Gamma(G, H)$ is still a regular graph of degree $|H|$. Let $G_{1}$ be the least subgroup of $G$ containing $H$. Then $H$ is a connecting set for $G_{1}$, and $\Gamma(G, H)$ is a disconnected graph isomorphic to the union of $\left|G: G_{1}\right|$ copies of $\operatorname{Cay}\left(G_{1}, H\right)$. In particular, the eigenvalues of $\Gamma(G, H)$ and $\operatorname{Cay}\left(G_{1}, H\right)$ are the same (disregarding the multiplicities). In fact, $A(G, H)$ is similar to a block diagonal matrix in which all blocks are $A\left(G_{1}, H\right)$. So we have: 
Lemma 2.7 Let $H$ be a subset of the group $G$ such that $1 \notin H$ and $H=H^{-1}$. Let $G_{1}$ be the smallest subgroup of $G$ containing $H$. Then $\Gamma(G, H)$ and $\operatorname{Cay}\left(G_{1}, H\right)$ have the same distinct eigenvalues.

Thus, such a generalization is not essential. However, for the study of eigenvalues of Cay $\left(G_{1}, H\right)$ by means of representation theory one sometimes prefers to deal with the adjacency matrix of $\Gamma(G, H)$ if the representation theory of $G$ is simpler than that of $G_{1}$. In Sect. 6, we observe this for $G=S_{n}$ and $G_{1}=A_{n}$.

There is no harm in identifying $g \in G$ with its matrix in the regular representation of $G$, of size $|G| \times|G|$. A key fact for adjacency matrices of Cayley graphs then is that $\sum_{h \in H} h$, the sum over $h \in H$ of the matrices just defined, is the adjacency matrix of $\operatorname{Cay}(G, H)$. Thus $A(G, H)=H^{+}$under this identification. (Recall that we define $H^{+}$as an element of the group algebra of $G$ over $\mathbb{C}$.) This is well known and explained, for instance, in [17, p. 384]. In other words, $A$ is the image of $H^{+}$in the regular representation of the group algebra. Note that $A$ is a linear transformation of the vector space $\mathbb{C} V=\mathbb{C} G$, the $\mathbb{C}$-span of $G$.

Lemma 2.8 Let $H$ be a connecting set in the group $G$ and let $F$ be the normalizer of $H$ in $G$. Then $F \times G$ acts as a group of automorphisms on $\Gamma=\operatorname{Cay}(G, H)$ by $(f, g): v \mapsto f^{-1} v g$ for all $(f, g) \in F \times G$ and $v \in G$. In particular, Aut $(\Gamma)$ is vertex transitive.

Proof Let $u, v \in G$. Then $u v^{-1} \in H$ if and only if $\left(f^{-1} u g\right)\left(g^{-1} v^{-1} f\right) \in f^{-1} H f=$ $H$. Evidently $\left\{1_{F}\right\} \times G$ is transitive on $G$.

\section{Some general results}

In this section we discuss representation theoretical aspects of eigenvalue problems for Cayley graphs. Let $G$ be a finite group, $H \subset G$ a connecting subset, and put $H^{+}=\sum_{h \in H}$ as an element in the group algebra $\mathbb{C} G$ of $G$ over $\mathbb{C}$. If $\rho$ is the regular representation of $G$ then $A(G, H)=\rho\left(H^{+}\right)$. If we identify $g$ with $\rho(g)$ then $A(G, H)=H^{+}$as discussed in Sect. 2.4. Thus, if $\phi$ is any representation of $G$ then $\phi\left(H^{+}\right)$is meaningful, and the eigenvalues of $\phi\left(H^{+}\right)$are eigenvalues of $\Gamma$. We think that this convention makes the exposition more transparent.

By standard results, $\rho$ is a direct sum of all irreducible representations $\phi$ of $G$, each occurring with multiplicity $\operatorname{dim} \phi$. It follows that the set of all eigenvalues of $\operatorname{Cay}(G, H)$ (disregarding the multiplicities) is the union, over the irreducible representations $\phi$ of $G$, of the eigenvalues of $\phi\left(H^{+}\right)$. See [11, Proposition 7.1] or elsewhere.

If $\phi_{0}$ is the trivial representation of $G$ then, obviously, $\phi_{0}\left(H^{+}\right)=|H|$. Conversely, if $|H|$ is an eigenvalue of $\phi\left(H^{+}\right)$, where $\phi$ is an irreducible representation of $G$, then $\phi=\phi_{0}$ by Theorem 2.1 (as $\phi_{0}$ occurs in $\rho$ with multiplicity 1 ; here we use that $H$ is a connecting set).

Lemma 3.1 Let $G_{1}$ be a subgroup of $G$ and let $M$ be an irreducible $G$-submodule with character $\chi$. Let $H$ be a subset of $G$. 
(1) If $H$ is $G$-stable (that is, $g \mathrm{Hg}^{-1}=H$ for every $g \in G$ ) then the restriction $H^{+}$to $M$ acts as a scalar matrix $\mu$. Id. If $H=h^{G}$ is a conjugacy class then $\mu=|H| \cdot \frac{\chi(h)}{\chi(1)}=\left|G: C_{G}(h)\right| \cdot \frac{\chi(h)}{\chi(1)}$.

(2) Let $L$ be an irreducible $G_{1}$-module occurring in $\left.M\right|_{G_{1}}$ exactly once.

(i) Suppose that $H$ is $G_{1}$-stable. Then $H^{+}$acts scalarly on $L$.

(ii) Suppose that $H=H_{1} \cup H_{2}$, where $H_{1}, H_{2}$ are $G_{1}$-stable. Then $H^{+}, H_{1}^{+}$and $H_{2}^{+}$ act scalarly on $L$, and $v=v_{1}+v_{2}$, where $v, v_{1}, v_{2}$ are the eigenvalues of $\mathrm{H}^{+}, \mathrm{H}_{1}^{+}$ and $\mathrm{H}_{2}^{+}$on $L$. This remains true if $H=H_{1} \cup \cdots \cup H_{k}$, where $H_{1}, \ldots, H_{k}$ are $G_{1}$-normal. (That is, each $H_{i}^{+}$acts scalarly on $L$, and $v=v_{1}+\cdots+v_{k}$, where $v_{i}$ is the eigenvalue of $H_{i}^{+}$on $L$.

(iii) Suppose that $H_{1}=H \cap G_{1}$ and that $H_{1}$ is a connecting set in $G_{1}$. Then $v_{1}$ is the eigenvalue of the Cayley graph $\operatorname{Cay}\left(G_{1}, H_{1}\right)$ occurring on L. In particular, if $H_{1}$ is a single conjugacy class of $G_{1}$ and $\chi_{L}$ is the character of $L$ then $v_{1}=\left|H_{1}\right| \cdot \frac{\chi_{L}\left(h_{1}\right)}{\chi_{L}(1)}$, where $h_{1} \in H_{1}$.

Proof (1) is well known. (2) (i) Clearly, $H^{+}$commutes with every $g \in G_{1}$. Therefore, $H^{+} L$ is a $G_{1}$-module. By assumption, $H^{+} L=L$. Then, by Schur's lemma, $H^{+}$acts scalarly on $L$.

(ii) By (2), $H_{i}^{+}$stabilizes $L$ and acts on it as $v_{i} \cdot \operatorname{Id}$ for $i=1,2$. As $H^{+}=H_{1}^{+}+H_{2}^{+}$, the claim follows.

(iii) Let $V_{1}=G_{1}$ be the vertices of the Cayley graph Cay $\left(G_{1}, H_{1}\right)$. As $L$ is an irreducible $G_{1}$-module, $L$ is an irreducible constituent of the regular $G_{1}$-module $\mathbb{C} V_{1}=\mathbb{C} G_{1}$, and hence the eigenvalue of $H_{1}^{+}$on $L$ occurs as an eigenvalue of $H_{1}^{+}$ on $\mathbb{C} G_{1}$. The second claim in (iii) follows from (1).

Remarks. (1) Unfortunately, $v_{1}$ and $v_{2}$ depend not only on $L$ but on both $M, L$. In other words, if $L$ is a constituent of $M^{\prime}$, an irreducible $G$-module not isomorphic to $M$, then $v_{1}(M, L)$ may not be equal to $v_{1}\left(M^{\prime}, L\right)$. However, if $H_{1}=H \cap G_{1}$ then $v_{1}$ depends on $L$ but does not depend on $M$.

(2) If $G=S_{n}, G_{1}=S_{n-1}$, then every irreducible $G$-module $M$ is multiplicity free as a $G_{1}$-module (that is, every irreducible constituent of $\left.M\right|_{S_{n-1}}$ occurs exactly once). This follows from the branching rule expressed in terms of Young diagrams (we cannot get the same diagram by removing distinct single boxes from a given Young diagram. This remains true for alternating groups.) In addition, if $C$ is a conjugacy class of $S_{n}$ then $C \cap S_{n-1}$ is either empty or a single conjugacy class of $G_{1}$, as two permutations $x, y \in G_{1}$ are conjugate in $S_{n}$ if and only if they are conjugate in $G_{1}$. In the following we view $\operatorname{Sym}(1, \ldots, k)$ as the largest subgroup of $S_{n}$ which fixes all $i \notin\{1, \ldots, k\}$, etc.

Lemma 3.2 Let $1 \leq r<n-1$ and $G=S_{n}$. Let $M$ be the natural permutation $G$ module and $F=\operatorname{Sym}(1, \ldots, r) \times \operatorname{Sym}(r+1, \ldots, n) \subset G$. Suppose that the subset $H \subset G$ is normalized by $F$, that is $x H x^{-1}=H$ for all $x \in F$. If $r \geq 2$, then $H^{+}$has at most 4 distinct eigenvalues on $M$. Two of these have multiplicity 1 , and two others, if distinct, have multiplicity $r-1$ and $n-r-1$. If $r=1$, then $H^{+}$has at most 3 distinct eigenvalues on $M$. Two of these have multiplicity 1, the remaining eigenvalue has multiplicity $n-2$. 
Proof Set $F_{1}=\operatorname{Sym}(1, \ldots, r) \times \operatorname{Id}$ and $F_{2}=\operatorname{Id} \times \operatorname{Sym}(r+1, \ldots, n)$. As $H$ is $F$ invariant, $H^{+}$commutes with $F$ in the group algebra $\mathbb{C} S_{n}$ and hence in End $M$. We have $\left.M\right|_{F}=M_{1} \oplus M_{2}$, where $M_{i}$ is an $F$-module such that $\left.M_{i}\right|_{F_{i}}$ is the natural permutation modules for $F_{i}$ and $\left.M_{i}\right|_{F_{j}}$ is trivial for $i \neq j, i, j=1,2$. Then $M_{1}=T_{1} \oplus L_{1}$, where $T_{1}$ is the trivial $F_{1}$-module and $L_{1}$ is irreducible of dimension $r-1$. In particular, $L_{1}=0$ when $r=1$. Similarly, $M_{2}=T_{2} \oplus L_{2}$, where $L_{2}$ is irreducible of dimension $n-r-1$. Clearly, $L_{1}, L_{2}$ are non-equivalent non-trivial $F$-modules. So $\left.M\right|_{F}=L_{1} \oplus L_{2} \oplus T$, where $T$ is a trivial $F$-module of dimension 2. By Schur's lemma, $H^{+}$acts scalarly on $L_{1}, L_{2}$, and hence $H^{+}$has at most four distinct eigenvalues on $M$. Clearly, if $r=1$ then $L_{0}=0$ and so $H^{+}$has at most three distinct eigenvalues.

\section{Symmetric group: the largest conjugacy classes}

For basic definitions concerning the characters of symmetric and alternating groups see $[8,9]$.

Let $G=S_{n}$ and let $1 \leq k \leq n$. We denote the set of all $k$-cycles in $G$ by $C(n, k)$. Since two permutations are conjugate to each other in $G$ if and only if they have the same cycle type we have $|C(n, k)|=\left|G: C_{G}(g)\right|$ when $g$ is any $k$-cycle and $C_{G}(g)$ denotes the centralizer of $g$ in $G$. In particular, $|C(n, n)|=(n-1)$ ! and $|C(n, n-1)|=n(n-2)$ !. By the same argument, the enumeration of permutations by cycle-type shows that all other conjugacy class sizes in $G$ are smaller if $n>4$. It is well-known that the smallest subgroup of $S_{n}$ containing $C(n, k)$ is $S_{n}$ if $k$ is even and $A_{n}$ if $k$ is odd.

In this section, we compute the second largest eigenvalue of $H^{+}$when $H=C(n, n)$ or $H=C(n, n-1)$. For this we first quote a well-known result for the cycles of length $n$ in representations of $S_{n}$ [8, Lemma 21.4]. Below let $\chi=\chi_{\mu}$ denote an irreducible character of $G$ labeled by the Young diagram $\mu$.

Lemma 4.1 Let $h \in S_{n}$ be an $n$-cycle. Then $\chi(h)=0$ if $\mu$ is not a hook. If $\mu=$ $\left[n-m, 1^{m}\right]$ is a hook with leg length $m$ then $\chi(h)=(-1)^{m}$.

Note that for $n \geq 4$ we have $\chi_{\left[n-2,1^{2}\right]}(1)=(n-1)(n-2) / 2$ and $\chi_{[n-2,2]}(1)=$ $n(n-3) / 2$, so $\chi_{[n-2,2]}(1)+1=\chi_{\left[n-2,1^{2}\right]}(1)$.

Lemma 4.2 Let $h \in S_{n}$ be an $n$-cycle, $n>4$, and let $H=h^{G}$ be the conjugacy class of $h$.

(1) Suppose that $n$ is odd. Then the maximum of the irreducible character ratio $\chi(h) / \chi(1)$ with $\chi(1) \neq 1$ is attained at $\chi_{\left[n-2,1^{2}\right]}$ and equals $2 /(n-1)(n-2)$. The corresponding eigenvalue of $H^{+}$equals $2|H| /(n-1)(n-2)=2(n-3)$ !.

(2) Suppose that $n$ is even. Then the maximum of the irreducible character ratio $\chi(h) / \chi(1)$ with $\chi(1) \neq 1$ is attained at $\chi_{\left[2,1^{n-2}\right]}$ and equals $1 /(n-1)$. The corresponding eigenvalue of $H^{+}$equals $|H| /(n-1)=(n-2)$ !

Proof By Lemma 3.1(1) it suffices to prove the claim on the ratio, and, by Lemma 4.1, only for the hook characters $\chi_{\left[n-m, 1^{m}\right]}$ with $m$ even. In this case $\chi_{\left[n-m, 1^{m}\right]}(h)=1$, so 
we have to show that $\chi_{\left[n-m, 1^{m}\right]}(1)$ is minimal for $m=2$ for $n$ odd and $n-2$ for $n$ even.

(1) Here $\chi_{\left[n-2,1^{2}\right]}(h)=1=\chi_{\left[3,1^{n-3}\right]}(h)$ and $\chi_{\left[n-2,1^{2}\right]}(1)=(n-1)(n-2) / 2=$ $\chi_{\left[3,1^{n-3}\right]}$. As $\chi_{[n-1,1]}(h)=\chi_{\left[2,1^{n-2}\right]}(h)=-1$, it suffices to show that $\chi_{\left[n-m, 1^{m}\right]}(1)>$ $(n-1)(n-2) / 2$ for $2<m<n-3$. For such $m$, we have

$$
\chi_{\left[n-m, 1^{m}\right]}(1)=\frac{n !}{n(n-m-1) ! m !}=\frac{(n-1)(n-2) \cdots(n-m+1)}{2 \cdot 3 \cdots \cdots m}>\frac{(n-1)(n-2)}{2} .
$$

(2) In this case $\chi_{[n-1,1]}(h)=-1, \chi_{\left[2,1^{n-2}\right]}(h)=1$ as these characters differ by sign character multiple. In addition, $\chi_{\left[2,1^{n-2}\right]}(1)=n-1<(n-1)(n-2) / 2<\chi_{\left[n-m, 1^{m}\right]}(1)$ for $3<m<n-4$. So the result follows.

Lemma 4.3 Let $h \in G=S_{n}, n>4$, be an $(n-1)$-cycle and let $H$ be the conjugacy class of $h$.

(1) Suppose $n$ is even. Then the maximum of the irreducible character ratio $\chi(h) / \chi(1)$ with $\chi(1) \neq 1$ is attained at $\chi_{[n-3,2,1]}$ and equals $3 / n(n-2)(n-4)$; the corresponding eigenvalue of $H^{+}$equals $3|H| / n(n-2)(n-4)=3(n-3)(n-5)$ !.

(2) Suppose $n$ is odd. Then the maximum of the irreducible character ratio $\chi(h) / \chi(1)$ with $\chi(1) \neq 1$ is attained at $\chi_{\left[2,2,1^{n-4}\right]}$ and equals $2 / n(n-3)$; the corresponding eigenvalue of $H^{+}$equals $2|H| / n(n-3)=2(n-2)(n-4)$ !.

Proof Note that $|H|=n ! /(n-1)=n(n-2)$ !. Let $\chi=\chi_{\mu}$ be an irreducible character of $G$ labeled by the Young diagram $\mu$. Suppose that $\chi(1)>1$. Let $h \in H$; we can assume that $h \in C_{G}(n) \cong S_{n-1}$.

(i) We have $\chi(h)=0$ unless $\mu=\left[n-m, 2,1^{m-2}\right]$ with $(2 \leq m \leq n-2)$. To show this let $\left.\chi\right|_{S_{n-1}}=v_{1}+\cdots+v_{k}$, where $v_{1}, \ldots, v_{k}$ are irreducible character of $S_{n-1}$; then $\chi(h)=v_{1}(h)+\cdots+v_{k}(h)$. By Lemma 4.1, $v_{i}(h)=0$ unless $v_{i}$ corresponds to a hook. So $\chi(h)=0$ unless $\mu$ is obtained from a hook diagram by adding one box. Obviously, either $\mu$ itself is a hook or $\mu$ is obtained from a hook diagram by adding one box at the (2,2)-position, that is, $\mu=\left[n-m, 2,1^{m-2}\right]$ for $m=2, \ldots, n-2$.

Suppose $\mu=\left[n-m, 1^{m}\right]$ is a hook, $1 \leq m \leq n-1$. Then $\left.\chi\right|_{S_{n-1}}=v_{1}+v_{2}$, where the diagrams of $v_{1}, v_{2}$ are $\left[n-m-1,1^{m}\right]$ and $\left[n-m, 1^{m-1}\right]$. The legs of these hooks are of distinct parity, so $v_{1}(h)+v_{2}(h)=0$ by Lemma 4.1 .

(ii) For $2 \leq m \leq n-2$ set $\chi_{m}=\chi_{\mu}$ for $\mu=\left[n-m, 2,1^{m-2}\right]$. Then $\chi_{\mu}(h)=$ $(-1)^{m-1}$.

Indeed, if $2<m<n-1$ then $\left.\chi_{m}\right|_{S_{n-1}}=v_{1}+v_{2}+v_{3}$, where $v_{1}, v_{2}, v_{3}$ are irreducible characters of $S_{n-1}$ with Young diagrams $\left[n-m-1,2,1^{m-2}\right]$, $\left[n-m, 2,1^{m-3}\right]$ and $\left[n-m, 1^{m-1}\right]$, respectively. So $v_{1}(g)=v_{2}(g)=0$ by Lemma 4.1, whereas $\nu_{3}(g)=(-1)^{m-1}$. So $\chi_{m}(h)=(-1)^{m-1}$. If $m=2$ then $\left.\chi_{2}\right|_{S_{n-1}}=v_{1}+v_{3}$, whose Young diagrams are $[n-3,2]$ and $[n-2,1]$. As above, $\nu_{1}(h)=0, v_{3}(h)=-1$ so $\chi_{2}(h)=-1$. Let $m=n-2$, so $\mu=\left[2,2,1^{n-4}\right]$. Then $\chi_{\left[2,2,1^{n-4}\right]}(h)=(-1)^{n} \chi_{[n-2,2]}(h)$ (because $h$ takes the value -1 at the sign non-trivial character if and only if the permutation $h$ is even). So the claim follows.

Thus, $\chi_{\mu}(h)=1$ if $\mu=\left[n-m, 2,1^{m-2}\right]$ with $m$ odd, and $\chi_{\mu}(h)<1$ otherwise (assuming $\chi_{\mu}(1) \neq 1$ ). Therefore, to complete the proof of the lemma it suffices to determine the minimum of $\chi_{\mu}(1): \mu=\left[n-m, 2,1^{m-2}\right], m$ odd. 
(iii) Let $2 \leq m \leq(n-1) / 2$. Then

$$
\begin{aligned}
\chi_{m}(1) & =\frac{n !}{(n-m-2) !(n-m)(n-1) m(m-2) !} \\
<\chi_{m+1}(1) & =\frac{n !}{(n-m-3) !(n-m-1)(n-1)(m+1)(m-1) !}
\end{aligned}
$$

as $(n-m-1)(m+1)(m-1)<m(n-m)(n-m-2)$. (Indeed, $m \leq(n-1) / 2$ implies $n-m \geq m+1$ and $m(n-m)(n-m-2) \geq(n-m-2) m(m+1)=$ $(n-m-1) m(m+1)-m(m+1) \geq(n-m-1) m(m+1)-(n-m-1)(m+1)=$ $(n-m-1)(m+1)(m-1)$.

(iv) By (ii) and (iii), the maximum of the ratio $\chi_{m}(h) / \chi_{m}(1)$ is attained for $m=n-2$ if $n$ is even, and for $n=3$ is $n$ is odd. This implies the result.

We expect that the behavior of $\chi_{[n-1,1]}(h)$ is not typical when $h$ is a $k$-cycle with $k=n, n-1$. Namely, for $k<n-1$ we expect that the second largest eigenvalue is attained for $\chi=\chi[n-1,1]$.

By Lemma 2.7 we have:

Lemma 4.4 For $n>4$, let $H$ be the set of all $n$-cycles in $A_{n}$ if $n>3$ is odd and all $n-1$-cycles in $A_{n}$ if $n>5$ is even. Then $H$ is a connecting set in $V=A_{n}$ and the largest second eigenvalue of $H^{+}$on the Cayley graph Cay $\left(A_{n}, H\right)$ equals $2(n-3)$ ! and $3(n-3)(n-5) !$, respectively.

Proof of Theorems 1.1 and 1.2 If $G=A_{n}$ the result follows from Lemma 4.4. If $G=$ $S_{n}$ then $\operatorname{Cay}(G, H)$ is bipartite, with partition $G=A_{n} \cup\left(S_{n} \backslash A_{n}\right)$, since $u \sim v$ implies that $u v^{-1} \in H \subset S_{n} \backslash A_{n}$ and so $u, v$ do not have the same parity. Hence $|H|$ and $-|H|$ are eigenvalues of $\operatorname{Cay}(G, H)$ by Theorem 2.1, corresponding to the trivial and the alternating characters of $S_{n}$, respectively. All other characters have degree $>1$ and so the result follows from Lemmas 4.2 and 4.3.

\section{The natural $S_{n}$-module and equitable partitions}

Let $G=S_{n}$ and let $K$ be the stabilizer of some point in $\{1, \ldots, n\}$. Then the set $G / K$ of cosets of $K$ in $G$ and $\{1, \ldots, n\}$ are isomorphic as $G$-sets. The associated permutation module over $\mathbb{C}$ is the natural module for $G$, denoted $M=1_{K}^{G}$. This is probably the most important $S_{n}$-module. Moreover, we expect that in many cases the second largest eigenvalue of a Cayley graph over $S_{n}$ occurs on $M$ (although we have no means to justify this).

For $1 \leq r<k \leq n$ let $C(n, k ; r) \subseteq C(n, k)$ be the set of all $k$ cycles in $S_{n}$ which move all the points in the set $\{1,2, \ldots, r\}$. That is to say, $g=\left(i_{1}, i_{2}, \ldots, i_{k}\right)\left(i_{k+1}\right) \ldots\left(i_{n}\right) \in C(n, k ; r)$ if and only if $\{1,2, \ldots, r\} \subset$ $\left\{i_{1}, i_{2}, \ldots, i_{k}\right\}$. Clearly, $|C(n, k ; r)|=(k-1) !\left(\begin{array}{c}n-r \\ k-r\end{array}\right)$.

Lemma 5.1 Let $1 \leq r<k \leq n$ and let $X$ be the smallest subgroup of $S_{n}$ containing $C(n, k ; r)$. Then $X=S_{n}$ if $k$ is even, and $X=A_{n}$ if $k$ is odd. 
Proof This holds when $k=n$ since $C(n, n ; r)=C(n, n)$ for all $r$ and so it suffices to show that $X \supseteq \mathrm{Alt}_{n}$ for $1 \leq r<k<n$. Since for every $i \in\{2, \ldots, n\}$ there is some $h \in C(n, k ; r)$ with $h(i)=1$ it follows that $X$ is transitive on $\{1,2, \ldots, n\}$. If $i<n$ we may assume additionally that $h(n)=n$ since $k<n$. Hence, the stabilizer of $n$ in $X$ is transitive on $\{1,2, \ldots, n-1\}$ and so $X$ is doubly transitive. The result follows if $k=2$ or $=3$, since a double transitive group containing a 2- or 3-cycle contains $\mathrm{Alt}_{n}$. For $k \geq 4$, consider the elements $h=(1,2, \ldots, k-3, k-2, k-1, k)$ and $h^{\prime}=(k-1, k, k-2, k-3, \ldots, 2,1)$. Then $h^{\prime} h=(1)(2) \ldots(k-3)(k-2, k, k-1) \in X$ is a 3 -cycle and hence the result follows.

Theorem 5.2 Let $G=S_{n}$ and $H=C(n, k ; r)$ for $2 \leq r<k<n$. Then the eigenvalues of $\mathrm{H}^{+}$on the natural module $M$ are

$$
\begin{aligned}
& \mu_{1}=|H|=(k-1) !\left(\begin{array}{c}
n-r \\
k-r
\end{array}\right), \quad \mu_{2}=(k-2) !\left(\begin{array}{c}
n-r \\
k-r
\end{array}\right) \frac{1}{n-r}\left((k-1)(n-k)-\frac{(k-r-1)(k-r)}{n-r-1}\right) \\
& \mu_{3}=(k-2) !\left(\begin{array}{c}
n-r \\
k-r
\end{array}\right)\left(\frac{r(n-k)}{n-r}-1\right) \text { and } \mu_{4}=-(k-2) !\left(\begin{array}{c}
n-r \\
k-r
\end{array}\right) .
\end{aligned}
$$

In the remainder of this section let $k, G$ and $H$ be as in the theorem and put $\Gamma=\operatorname{Cay}(G, H)$. We exhibit two equitable partitions of $\Gamma$ so that their associated eigenvalues belong to $\left\{\mu_{1}, \mu_{2}, \mu_{3}, \mu_{4}\right\}$. The proof is completed at the end of the section.

First let $1 \leq r$ and let $K_{1}=K=\operatorname{Sym}(1, \ldots, n-1)$ be the stabilizer in $G$ of $n$. Define the partition $P_{1}$ of the vertices of $\Gamma$ by

$$
\begin{aligned}
& V_{1}=K=\{g \in G: g(n)=n\} ; \\
& V_{2}=(n, 1) K \cup \cdots \cup(n, r) K=\{g \in G: g(n) \in\{1, \ldots, r\}\} ; \\
& V_{3}=(n, r+1) K \cup \cdots \cup(n, n-1) K=\{g \in G: g(n) \in\{r+1, \ldots, n-1\}\} .
\end{aligned}
$$

Below $\left(\begin{array}{l}a \\ b\end{array}\right)$ for integers $a \geq b>0$ denotes the number of choices of $b$ elements from a set of $a$ elements. For uniformity, we need to make the cases with $b=0$ and $b=-1$ meaningful: we define $\left(\begin{array}{l}a \\ 0\end{array}\right)$ to be 1 , and $\left(\begin{array}{c}a \\ -1\end{array}\right)$ to be 0 .

Lemma 5.3 Let $1 \leq r<k<n$. Then $P_{1}$ is an equitable partition of $\Gamma$ with matrix

$$
\begin{aligned}
& B_{1}=\left(\begin{array}{ccc}
(k-1) !\left(\begin{array}{c}
n-r-1 \\
k-r
\end{array}\right) & r(k-2) !\left(\begin{array}{c}
n-r-1 \\
k-r-1
\end{array}\right) & (n-r-1)(k-2) !\left(\begin{array}{c}
n-r-2 \\
k-r-2
\end{array}\right) \\
(k-2) !\left(\begin{array}{c}
n-r-1 \\
k-r-1
\end{array}\right) & (r-1)(k-2) !\left(\begin{array}{c}
n-r \\
k-r
\end{array}\right) & (n-r-1)(k-2) !\left(\begin{array}{c}
n-r-1 \\
k-r-1
\end{array}\right) \\
(k-2) !\left(\begin{array}{c}
n-r-2 \\
k-r-2
\end{array}\right) & r(k-2) !\left(\begin{array}{c}
n-r-1 \\
k-r-1
\end{array}\right) & (n-r-2)(k-2) !\left(\begin{array}{c}
n-r-2 \\
k-r-2
\end{array}\right)+(k-1) !\left(\begin{array}{c}
n-r-1 \\
k-r
\end{array}\right)
\end{array}\right) \\
& =(k-2) !\left(\begin{array}{ccc}
(k-1)\left(\begin{array}{c}
n-r-1 \\
k-r
\end{array}\right) & r\left(\begin{array}{c}
n-r-1 \\
k-r-1
\end{array}\right) & (k-r-1)\left(\begin{array}{c}
n-r-1 \\
k-r-1
\end{array}\right) \\
\left(\begin{array}{c}
n-r-1 \\
k-r-1
\end{array}\right) & (r-1)\left(\begin{array}{c}
n-r \\
k-r
\end{array}\right) & (n-r-1)\left(\begin{array}{c}
n-r-1 \\
k-r-1
\end{array}\right) \\
\left(\begin{array}{c}
n-r-2 \\
k-r-2
\end{array}\right) & r\left(\begin{array}{c}
n-r-1 \\
k-r-1
\end{array}\right) & (n-r-2)\left(\begin{array}{c}
n-r-2 \\
k-r-2
\end{array}\right)+(k-1)\left(\begin{array}{c}
n-r-1 \\
k-r
\end{array}\right)
\end{array}\right) .
\end{aligned}
$$

The eigenvalues of $B_{1}$ are $\mu_{1}=(k-1) !\left(\begin{array}{c}n-r \\ k-r\end{array}\right)$,

$$
\begin{aligned}
& \mu_{2}=(k-2) !\left(\begin{array}{c}
n-r \\
k-r
\end{array}\right) \frac{1}{n-r}\left((k-1)(n-k)-\frac{(k-r-1)(k-r)}{n-r-1}\right) \text { and } \\
& \mu_{3}=(k-2) !\left(\begin{array}{c}
n-r \\
k-r
\end{array}\right)\left(r-1-r \frac{k-r}{n-r}\right)=(k-2) !\left(\begin{array}{c}
n-r \\
k-r
\end{array}\right)\left(\frac{r(n-k)}{n-r}-1\right) .
\end{aligned}
$$


Proof Put $F=\operatorname{Sym}(1, \ldots, r) \times \operatorname{Sym}(r+1, \ldots, n-1)$ and let $F \times K$ act on $V$ as in Lemma 2.8. Then $F \times K$ stabilizes each $V_{i}$ and acts transitively on it. Hence $P_{1}$ is equitable by Lemma 2.3 .

Let $B_{1}=\left(\beta_{i j}\right)$ for $1 \leq i, j \leq 3$ and so $\beta_{i j}=\left|N\left(v_{i}\right) \cap V_{j}\right|=\left|H v_{i} \cap V_{j}\right|=$ $\left|H \cap V_{j} v_{i}^{-1}\right|$ for $v_{i} \in V_{i}$. Taking $v_{1}=1_{G}, v_{2}=(n, 1)$ and $v_{3}=(n, n-1)$ we have $(i=1): \beta_{11}=|H \cap K|=(k-1) !\left(\begin{array}{c}n-r-1 \\ k-r\end{array}\right)$;

$\beta_{12}=\left|H \cap V_{2}\right|=r\left|H \cap K v_{2}\right|=r(k-2) !\left(\begin{array}{c}n-r-1 \\ k-r-1\end{array}\right)$;

$\beta_{13}=\left|H \cap V_{3}\right|=(n-r-1)\left|H \cap K v_{3}\right|=(n-r-1)(k-2) !\left(\begin{array}{l}n-r-2 \\ k-r-2\end{array}\right)$.

$(i=2): \beta_{21}=|H \cap K(n, 1)|=|\{h \in H: h(1)=n\}|=(k-2) !\left(\begin{array}{l}n-r-1 \\ k-r-1\end{array}\right)$;

$\beta_{22}=\left|H \cap V_{2} v_{2}\right|=\left|H \cap(n, 1) K v_{2}\right|+\left|H \cap(n, 2) K v_{2}\right|+\cdots+\left|H \cap(n, r) K v_{2}\right|$ $=0+(r-1)\left|(n, 2) K v_{2} \cap H\right|=(r-1)(k-2) !\left(\begin{array}{c}n-r \\ k-r\end{array}\right)$;

$\beta_{23}=\left|H \cap V_{3} v_{2}\right|=|H \cap(n, r+1) K(n, 1)|+\cdots+|H \cap(n, n-1) K(n, 1)|$ $=(n-r-1)(k-2 !)\left(\begin{array}{c}n-r-1 \\ k-r-1\end{array}\right)$.

$(i=3): \beta_{31}=\left|H \cap V_{1} v_{3}\right|=(k-2) !\left(\begin{array}{c}n-r-2 \\ k-r-2\end{array}\right)$;

$\beta_{32}=\left|H \cap V_{2} v_{3}\right|=r(k-2) !\left(\begin{array}{c}n-r-1 \\ k-r-1\end{array}\right)$;

$\beta_{33}=\left|H \cap V_{3} v_{3}\right|=(n-r-2)(k-2) !\left(\begin{array}{c}n-r-2 \\ k-r-2\end{array}\right)+(k-1) !\left(\begin{array}{c}n-r-1 \\ k-r\end{array}\right)$.

This gives the matrix above after some further transformations. We have confirmed the eigenvalues using a symbolic calculator such as Wolfram|Alpha [19].

Next let $2 \leq r$ and let $K_{2}=K=\operatorname{Sym}(2, \ldots, n)$ be the stabilizer in $G$ of 1 . Define the partition $P_{2}$ of the vertices of $\Gamma$ by

$$
\begin{aligned}
& V_{1}=K=\{g \in G: g(1)=1\} \\
& V_{2}=(2,1) K \cup \cdots \cup(r, 1) K=\{g \in G: g(1) \in\{2, \ldots, r\}\} \\
& V_{3}=(r+1,1) K \cup \cdots \cup(n, 1) K=\{g \in G: g(1) \in\{r+1, \ldots, n\}\}
\end{aligned}
$$

Lemma 5.4 Let $2 \leq r<k<n$. Then $P_{2}$ is an equitable partition of $\Gamma$ with matrix

$$
\begin{aligned}
B_{2} & =\left(\begin{array}{ccc}
0 & (r-1)(k-2) !\left(\begin{array}{c}
n-r \\
k-r
\end{array}\right) & (k-r)(k-2) !\left(\begin{array}{c}
n-r \\
k-r
\end{array}\right) \\
(k-2) !\left(\begin{array}{c}
n-r \\
k-r
\end{array}\right) & (k-2) !(r-2)\left(\begin{array}{c}
n-r \\
k-r
\end{array}\right) & (k-r)(k-2) !\left(\begin{array}{c}
n-r \\
k-r
\end{array}\right) \\
(k-2) !\left(\begin{array}{c}
n-r-1 \\
k-r-1
\end{array}\right) & (r-1)(k-2) !\left(\begin{array}{c}
n-r \\
k-r-1
\end{array}\right)(k-2) !(n-r-1)\left(\begin{array}{c}
n-r-2 \\
k-r-2
\end{array}\right)+(k-1) !\left(\begin{array}{c}
n-r-1 \\
k-r
\end{array}\right)
\end{array}\right) \\
= & (k-2) !\left(\begin{array}{c}
n-r \\
k-r
\end{array}\right)\left(\begin{array}{ccc}
0 & r-1 & k-r \\
1 & r-2 & k-r \\
\frac{k-r}{n-r} & (r-1) \frac{k-r}{n-r} & k-1-r \frac{(k-r)}{n-r}
\end{array}\right) .
\end{aligned}
$$

The eigenvalues of $B_{2}$ are $\mu_{1}=(k-1) !\left(\begin{array}{l}n-r \\ k-r\end{array}\right), \mu_{3}=(k-2) !\left(\begin{array}{l}n-r \\ k-r\end{array}\right)\left(\frac{r(n-k)}{n-r}-1\right)$ and $\mu_{4}=-(k-2) !\left(\begin{array}{c}n-r \\ k-r\end{array}\right)$. 
Proof Put $F=\operatorname{Sym}(2, \ldots, r) \times \operatorname{Sym}(r+1, \ldots, n)$ and let $F \times K$ act on $V$ as in Lemma 2.8. Then $F \times K$ stabilizes each $V_{i}$ and acts transitively on it. Hence $P_{1}$ is equitable by Lemma 2.3 .

Let $B_{2}=\left(\beta_{i j}\right)$ for $1 \leq i, j \leq 3$ and so $\beta_{i j}=\left|N\left(v_{i}\right) \cap V_{j}\right|=\left|H v_{i} \cap V_{j}\right|=$ $\left|H \cap V_{j} v_{i}^{-1}\right|$ for $v_{i} \in V_{i}$. Taking $v_{1}=1_{G}, v_{2}=(2,1)$ and $v_{3}=(n, 1)$ we have

$(i=1): \beta_{11}=|H \cap K|=0$;

$\beta_{12}=\left|H \cap V_{2}\right|=(r-1)(k-2) !\left(\begin{array}{c}n-r \\ k-r\end{array}\right)$;

$\beta_{13}=\left|H \cap V_{3}\right|=(n-r)(k-2) !\left(\begin{array}{c}n-r-1 \\ k-r-1\end{array}\right)=(k-r)(k-2) !\left(\begin{array}{c}n-r \\ k-r\end{array}\right)$.

$(i=2): \beta_{21}=|H \cap K(12)|=(k-2) !\left(\begin{array}{c}n-r \\ k-r\end{array}\right)$;

$\beta_{22}=\left|H \cap V_{2}(1,2)\right|=(r-2)(k-2) !\left(\begin{array}{c}n-r \\ k-r\end{array}\right)$;

$\beta_{23}=\left|H \cap V_{3}(1,2)\right|=(n-r)(k-2) !\left(\begin{array}{c}n-r-1 \\ k-r-1\end{array}\right)=(k-r)(k-2) !\left(\begin{array}{c}n-r \\ k-r\end{array}\right)$.

$(i=3): \beta_{3,1}=|H \cap K(1, n)|=(k-2) !\left(\begin{array}{c}n-r-1 \\ k-r-1\end{array}\right)$;

$\beta_{32}=\left|H \cap V_{2}(1, n)\right|=(k-2) !(r-1)\left(\begin{array}{c}n-r-1 \\ k-r-1\end{array}\right)$;

$\beta_{33}=\left|H \cap V_{3}(1, n)\right|=(k-2) !(n-r-1)\left(\begin{array}{c}n-r-2 \\ k-r-2\end{array}\right)+(k-1) !\left(\begin{array}{c}n-r-1 \\ k-r\end{array}\right)$

$=(k-2) !(k-r-1)\left(\begin{array}{c}n-r-1 \\ k-r-1\end{array}\right)+(k-1) !\left(\begin{array}{c}n-r-1 \\ k-r\end{array}\right)$.

This gives the matrix above after some further transformations. We have confirmed the eigenvalues using a symbolic calculator.

We emphasize that $P_{1}$ and $P_{2}$ are equitable partitions of $V=G$ and that both are refined by the coset partition $P_{0}=g_{1} K \cup g_{2} K \cup \cdots \cup g_{n} K$ which gives rise to the natural module $M$. Therefore, by Lemma 2.2, all eigenvalues $\mu_{i}$ for $1 \leq i \leq 4$ in Theorem 5.2 are eigenvalues of $\operatorname{Cay}(G, H)$ for $H=C(n, k ; r)$.

Proof of Theorem 5.2. Let $1 \leq r<k<n, H=C(n, k ; r) \subset \operatorname{Sym}(1, \ldots, n)$ and let $\mu_{1}, \mu_{2}, \mu_{3}, \mu_{4}$ be the eigenvalues stated in Lemmas 5.3 and 5.4. By Lemma 2.2 we have $\mu_{1}=\lambda_{1}(\Gamma)$. Since $G / K_{1}$ and $G / K_{2}$ are permutationally isomorphic, by Lemma 2.2, $\mu_{1}, \mu_{2}, \mu_{3}, \mu_{4}$ are distinct eigenvalue of $H^{+}$on $M$. Next we note that $H$ is invariant under conjugation by $F=\operatorname{Sym}(1, \ldots, r) \times \operatorname{Sym}(r+1, \ldots, n)$. By Lemma 3.2, $\mathrm{H}^{+}$has at most four eigenvalues and hence the result.

Proof of Theorem 1.3 By the comment above the theorem follows from Theorem 5.2 and Lemma 2.4.

\section{Proof of Theorem 1.4}

In this section let $4<n, 2 \leq r \leq n-2$ and $H=C(n, r+1 ; r) \subset S_{n}$. Let $M$ be the natural $S_{n}$-module. 
Lemma 6.1 The multiplicities of the eigenvalues of $\mathrm{H}^{+}$on $\mathrm{M}$ are as follows:

\begin{tabular}{|c|c|c|c|c|}
\hline Eigenvalue of $H^{+}$on $M$ & $r !(n-r)$ & $r !(n-r-1)$ & $(r-1)(r-1) !(n-r-1)-r !$ & $-(r-1) !(n-r)$ \\
\hline the multiplicity & 1 & $n-r-1$ & 1 & $r-1$ \\
\hline
\end{tabular}

Proof The eigenvalues of $\left|H^{+}\right|$on $M$ are determined in Theorem 5.2, where we take $k=r+1$. Note that the trace of $h \in H$ on $M$ equals $n-r-1$. So the trace of $H^{+}$equals $\left|H^{+}\right|(n-r-1)=r !(n-r)(n-r-1)$. We know that the eigenvalue $\left|H^{+}\right|$has multiplicity 1 (Theorem 2.1), and the multiplicities $x, y, z$ of the three other eigenvalues are in the set $\{1, n-r-1, r-1\}$ by Lemma 3.2. So we have

$$
\begin{aligned}
& \left|H^{+}\right|(n-r-1)=r !(n-r)(n-r-1) \\
& \quad=r !(n-r)+x r !(n-r-1)+y((r-1)(r-1) !(n-r-1)-r !)-z(r-1) !(n-r) .
\end{aligned}
$$

If we take $x=n-r-1, y=1, z=r-1$ then the equality holds. In addition, the equality fails for any other choice of $\{x, y, z\} \in\{1, n-r-1, r-1\}$. Whence the result.

Corollary 6.2 Let $M^{\prime}$ be an $S_{n}$-module whose composition factor dimensions are at most $n-1$. Then the second largest eigenvalue of $\mathrm{H}^{+}$on $\mathrm{M}^{\prime}$ does not exceed $r !(n-$ $r-1)$.

Proof Let $\rho$ be an irreducible characters of $S_{n}$ of degree at most $n-1$. Then $\rho$ is well known to be a constituent either of $M$ or $M \otimes \sigma$, where $\sigma$ denotes the sign representation of $S_{n}$. It follows that the eigenvalues of $H^{+}$on $M^{\prime}$ are in the list of Lemma 3.2 if $r$ is even, and also their negatives, if $r$ is odd. So the result follows as $r !(n-r-1)>(r-1) !(n-r)$.

Lemma 6.3 Let $G=S_{n}$ and let L be an irreducible $G$-module. Then there is a basis of $L$ such that the matrices $g+g^{-1}(g \in G)$ are symmetric. Consequently, if $H \subset G$ is a subset such that $\mathrm{H}=\mathrm{H}^{-1}$ then the matrix of $\mathrm{H}^{+}$on $\mathrm{L}$ is symmetric.

Proof It is well known that there is a basis of $L$ such that all matrices $g \in G$ are orthogonal, that is, if $g_{L}$ is the matrix of $g$ on $L$ then $g_{L}^{T}=\left(g_{L}\right)^{-1}=\left(g^{-1}\right)_{L}$, where $\left(g_{L}\right)^{T}$ is the transpose of $g_{L}$. Then $g_{L}+g_{L}^{-1}=g_{L}+\left(g_{L}\right)^{T}$, whence the claim.

Lemma 6.4 Let L be an irreducible $S_{n}$-module and $\lambda$ an eigenvalue of $H^{+}$on L. Then $\lambda \leq|H|$ and $\lambda=|H|$ implies $\operatorname{dim} L=1$.

Proof If $H$ is connecting then the lemma follows from Theorem 2.1. Indeed, $\lambda=|H|$ if $L$ is the trivial module, and the trivial module has multiplicity 1 in $\mathbb{C} S_{n}$. If $H$ is not a connecting set then $H$ is a connecting set of $A_{n}$ (Lemma 5.1), and the same argument is valid if $L$ is an $A_{n}$-module. So $|H|$ is an eigenvalue of $H^{+}$on $L$ if and only if the restriction of $L$ to $A_{n}$ contains the trivial $A_{n}$-module. This is well known to imply that $\operatorname{dim} L=1$.

Set $H_{n-1}=H \cap K$, where $K=\operatorname{Sym}(1, \ldots, n-1)$ and $E=H \backslash H_{n-1} \subset$ $\operatorname{Sym}(1, \ldots, n-2, n)$. Then $\left|H_{n-1}\right|=r !(n-r-1)$ and $|E|=|H|-\left|H_{n-1}\right|=r$ !

Theorem 6.5 Let L be an irreducible $S_{n}$-module and $\operatorname{dim} L>n-1$. Let $\lambda$ be an eigenvalue of $H^{+}$on L. Then $\lambda \leq r !(n-r-1)$. 
Lemma 6.6 Theorem 6.5 is true for $r=n-2>2$.

Proof The case of $n=5$ can be checked straightforwardly. Let $n>5$.

Note that $r$ ! $(n-r-1)$ equals $(n-2)$ ! for $r=n-2$. We have $H_{n-1}=\{(n-$ $\left.\left.1, i_{1}, \ldots, i_{n-2}\right)\right\}$ and $E=\left\{\left(n, i_{1}, \ldots, i_{n-2}\right)\right\}$, where $\left\{i_{1}, \ldots, i_{n-2}\right\}=\{1, \ldots, n-2\}$. So $H_{n-1}$ is a conjugacy class in $\operatorname{Sym}\{1, \ldots, n-1\}$. Let $\alpha_{1}, \beta_{1}$ be the maximum of eigenvalues of $H_{n-1}^{+}, E^{+}$, respectively, on $L$. By the branching rule, as an $S_{n-1}$-module $L$ has no irreducible constituent of dimension 1 . So, by Lemma 4.2, if $n-1>4$ is even then $\alpha_{1} \leq(n-3)$ !, and if $n-1$ is odd then this is at most $\alpha_{1} \leq 2(n-4)$ !. As $E$ is a conjugacy class of $\operatorname{Sym}(1, \ldots, n-2, n) \cong S_{n-1}$, the same conclusion holds for the eigenvalue $\beta_{1}$ of $E^{+}$and $\alpha_{1}=\beta_{1}$. By Lemma 6.3, we can assume that the matrices of $H^{+}, H_{n-1}^{+}, E$ on $L$ are symmetric. If $\gamma_{1}$ is the largest eigenvalue of $H^{+}$on $L$, then, by the Weyl inequality, $\gamma_{1} \leq \alpha_{1}+\beta_{1}=2 \alpha_{1}$. So $\gamma_{1} \leq 4(n-4)$ ! if $n-1$ is odd, and $\gamma_{1} \leq 2(n-3)$ ! if $n-1$ is even. Obviously, $\gamma_{1}<(n-2)$ !.

Proof of Theorem 6.5 As mentioned above, as an $S_{n-1}$-module $L$ has no composition factor of dimension 1. Therefore, by Lemma 6.4, $H_{n-1}^{+}$does not have eigenvalue $\left|H_{n-1}^{+}\right|=r !(n-r-1)$ on $L$. We use induction on $n-r$ with $n-r=2$ as base of induction. The case with $r=n-2$ is settled in Lemma 6.6. By the induction assumption, if $\mu$ is an eigenvalue of $H_{n-1}^{+}$on $L$ then $\mu \leq r !(n-r-2)$.

Let $v$ be the largest eigenvalue of $E^{+}$on $L$. Then $v \leq|E|=r$ ! (by Theorem 2.1 applied to the graph $(E, V)$.) By the Weyl inequality (4) applied to $L$, we have $\lambda \leq \mu+v \leq r !(n-r-2)+r !=r !(n-r-1)$, as required.

Proof of Theorem 1.4 If $G=S_{n}$ then this is equivalent to Theorem 6.5. For $A_{n}$ the result follows from the statement for $S_{n}$ and Lemma 2.7 .

Acknowledgements We wish to thank the referees for their comments and for bringing other papers on this topic to our attention.

Final Note We would like to mention the recent asymptotic results by O. Parzanchevski \& Puder [15] and R. Maleki \& A. S. Razafimahatratra [14] relating to Theorems 1.1 and 1.2. Furthermore, recently X. Huang, Q. Huang \& S. M. Cioabă [7] have computed the second largest eigenvalue of some Cayley graphs for highly transitive permutation groups on the natural permutation module. One of the eigenvalues in Theorem 5.2 can also be determined by [7, Lemma 11].

Data Availability This documents has no associated data.

Open Access This article is licensed under a Creative Commons Attribution 4.0 International License, which permits use, sharing, adaptation, distribution and reproduction in any medium or format, as long as you give appropriate credit to the original author(s) and the source, provide a link to the Creative Commons licence, and indicate if changes were made. The images or other third party material in this article are included in the article's Creative Commons licence, unless indicated otherwise in a credit line to the material. If material is not included in the article's Creative Commons licence and your intended use is not permitted by statutory regulation or exceeds the permitted use, you will need to obtain permission directly from the copyright holder. To view a copy of this licence, visit http://creativecommons.org/licenses/by/4.0/.

\section{References}

1. Alon, N.: Eigenvalues and expanders. Combinatorica 6, 83-96 (1986) 
2. Barrett, W.: Hermitian and Positive Definite Matrices, Chapter 8 in: Handbook of Linear Algebra, Chapman and Hall, London (2007)

3. Brouwer, A.E., Haemers, W.H.: Spectra of Graphs. Springer, Berlin (2012)

4. Dembowski, P.: Finite Geometries. Springer Verlag, Berlin (1968)

5. Hoory, S., Linial, N., Wigderson, A.: Expander graphs and their applications. Bull. Amer. Math. Soc. 43, 439-561 (2006)

6. Huang, X., Huang, Q.: The second largest eigenvalue of some Cayley graphs on alternating groups. J. Algebraic Combin. 50, 99-111 (2019)

7. Huang, X., Huang, Q., Cioabă, S.M.: The second eigenvalue of some normal Cayley graphs of highly transitive groups. Electron. J. Combin. 26 (2) (2019)

8. James, G.: The Representation Theory of the Symmetric Groups. Springer, Berlin (1978)

9. James, G., Kerber, A.: The Representation Theory of the Symmetric Group. Addison-Wesley, London (1981)

10. Kalpakis, K., Yesha, Y.: On the bisection width of the transposition network. Networks 29, 69-76 (1997)

11. Krebs, M., Shaheen, A.: Expander Families and Cayley Graphs. Oxford University Press, Oxford (2011)

12. Liu, X., Zhou, S.: Eigenvalues of Cayley graphs. arXiv:1809.09829v2 [math.CO] 22 Jan 2019

13. Lubotzky, A.: Cayley Graphs: Eigenvalues, expander and random walks. In: Rowlinson, P. (Eds.) Surveys in Combinatorics BCC. LMS Mathematical Lecture Notes, 218, pp. 155-191 (1995)

14. Maleki, R., Razafimahatratra, A.S.: On the second eigenvalue of a Cayley graph of the symmetric group. arXiv: 2108.13585 [math.CO]

15. Parzanchevski, O., Puder, D.: Aldous's spectral gap conjecture for normal sets. Trans. Amer. Math. Soc. 373, $7067-7086(2020)$

16. Stanic, Z.: Inequalities for Graph Eigenvalues, LMS Lecture Note Series, vol. 423. Cambridge University Press, Cambridge (2015)

17. Siemons, J., Zalesski, A.E.: Remarks on singular Cayley graphs and vanishing elements of simple groups. J. Algebraic Combin. 50, 379-401 (2019)

18. Weyl, H.: Das asymptotische Verteilungsgesetz der Eigenwerte linearer partieller Differentialgleichungen. Math. Ann. 71, 441-479 (1912)

19. Wolfram|Alpha. https://www.wolframalpha.com/widgets/gallery/view.jsp?id=9aa01caf50c9307e9dabe $159 \mathrm{c} 9068 \mathrm{c} 41$

Publisher's Note Springer Nature remains neutral with regard to jurisdictional claims in published maps and institutional affiliations. 